\title{
Two complete and minimal systems associated with the zeros of the Riemann zeta function
}

\author{
par JEAN-FrANÇOIS BURNOL
}

\begin{abstract}
RÉSUMÉ. Nous relions trois thèmes restés jusqu'alors distincts: les propriétés hilbertiennes des zéros de Riemann, la "formule duale de Poisson" de Duffin-Weinberger (que nous appelons formule de co-Poisson), les espaces de fonctions entières "de Sonine" définis et étudiés par de Branges. Nous déterminons dans quels espaces de Sonine (étendus) les zéros forment un système complet, ou minimal. Nous obtenons des résultats généraux concernant la distribution des zéros des fonctions entières de de BrangesSonine. Nous attirons l'attention sur certaines distributions liées à la transformation de Fourier et qui sont apparues dans nos travaux antérieurs.
\end{abstract}

\begin{abstract}
We link together three themes which had remained separated so far: the Hilbert space properties of the Riemann zeros, the "dual Poisson formula" of Duffin-Weinberger (also named by us co-Poisson formula), and the "Sonine spaces" of entire functions defined and studied by de Branges. We determine in which (extended) Sonine spaces the zeros define a complete, or minimal, system. We obtain some general results dealing with the distribution of the zeros of the de-Branges-Sonine entire functions. We draw attention onto some distributions associated with the Fourier transform and which we introduced in our earlier works.
\end{abstract}

\section{The Duffin-Weinberger "dualized" Poisson formula (aka co-Poisson)}

We start with a description of the "dualized Poisson formula" of Duffin and Weinberger $([13,14])$. We were not aware at the time of $[7]$ that the formula called by us co-Poisson formula had been discovered (much) earlier. Here is a (hopefully not too inexact) brief historical account: the story starts with Duffin who gave in an innovative 1945 paper [10] a certain formula constructing pairs of functions which are reciprocal under the sine transform. As pointed out by Duffin in the conclusion of his paper a special instance of the formula leads to the functional equation of the $L$-function

Manuscrit reçu le 28 janvier 2003. 
$1-\frac{1}{3^{s}}+\frac{1}{5^{s}}-\ldots$ (as we explain below, this goes both ways in fact). The co-Poisson formula which we discuss later will stand in a similar relation with the zeta function $1+\frac{1}{2^{s}}+\frac{1}{3^{s}}+\ldots$, the pole of zeta adding its own special touch to the matter. Weinberger extended in his dissertation [25] this work of Duffin and also he found analogous formulae involving Hankel transforms. Boas [1] gave a formal argument allowing to derive Duffin type formulae from the Poisson formula. However formal arguments might be misleading and this is what happened here: formula [1, 3.(iii)] which is derived with the help of a purely formal argument looks like it is the co-Poisson formula, but is not in fact correct. It is only much later in 1991 that Duffin and Weinberger [13] (see also [14]) published and proved the formula which, in hindsight, we see now is the one to be associated with the Riemann zeta function. They also explained its "dual" relation to the so-much-well-known Poisson summation formula. In [7] we followed later a different (esoterically adelic) path to the same result. As explained in [7], there are manifold ways to derive the co-Poisson formula (this is why we use "co-Poisson" rather than the "dualized Poisson" of Duffin and Weinberger). In this Introduction we shall explain one such approach: a re-examination of the Fourier meaning of the functional equation of the Riemann zeta function.

When applied to functions which are compactly supported away from the origin, the co-Poisson formula creates pairs of cosine-tranform reciprocal functions with the intriguing additional property that each one of the pair is constant in some interval symmetrical around the origin. Imposing two linear conditions we make these constants vanish, and this leads us to a topic which has been invented by de Branges as an illustration, or challenge, to his general theory of Hilbert spaces of entire functions ([3]), apparently with the aim to study the Gamma function, and ultimately also the Riemann zeta function. The entire functions in these specific de Branges spaces are the Mellin transforms, with a Gamma factor, of the functions with the vanishing property for some general Hankel transform (the cosine or sine transforms being special cases). These general "Sonine Spaces" were introduced in [2], and further studied and axiomatized by J. and V. Rovnyak in [22]. Sonine himself never dealt with such spaces, but in a study ([23]) of Bessel functions he constructed a pair of functions vanishing in some interval around the origin and reciprocal under some Hankel transform. An account of the Sonine spaces is given in a final section of [3], additional results are to be found in [4] and [5]. As the co-Poisson formula has not been available in these studies, the way we have related the Riemann zeta function to the Sonine spaces in [7] has brought a novel element to these developments, a more intimate, and explicit, web of connections between 
the Riemann zeta function and the de Branges spaces, and their extensions allowing poles.

Although this paper is mostly self-contained, we refer the reader to "On Fourier and Zeta(s)" ([7]) for the motivating framework and additional background and also to our Notes $[6,8,9]$ for our results obtained so far and whose aim is ultimately to reach a better understanding of some aspects of the Fourier Transform.

Riemann sums $\sum_{n \geq 1} F(n)$, or $\sum_{n \geq 1} \frac{1}{T} F\left(\frac{n}{T}\right)$, have special connections with, on one hand the Riemann zeta function $\zeta(s)=\sum_{n \geq 1} \frac{1}{n^{s}}$ (itself obtained as such a summation with $F(x)=x^{-s}$ ), and, on the other hand, with the Fourier Transform.

In particular the functional equation of the Riemann zeta function is known to be equivalent to the Poisson summation formula:

$$
\sum_{n \in \mathbb{Z}} \widetilde{\phi}(n)=\sum_{m \in \mathbb{Z}} \phi(m)
$$

which, for simplicity, we apply to a function $\phi(x)$ in the Schwartz class of smooth quickly decreasing functions.

Note 1. We shall make use of the following convention for the Fourier Transform:

$$
\mathcal{F}(\phi)(y)=\widetilde{\phi}(y)=\int_{\mathbb{R}} \phi(x) e^{2 \pi i x y} d x
$$

With a scaling-parameter $u \neq 0,(1)$ leads to:

$$
\sum_{n \in \mathbb{Z}} \widetilde{\phi}(n u)=\sum_{m \in \mathbb{Z}} \frac{1}{|u|} \phi\left(\frac{m}{u}\right)
$$

which, for the Gaussian $\phi(x)=\exp \left(-\pi x^{2}\right)$, gives the Jacobi identity for the theta function (a function of $u^{2}$ ). Riemann obtains from the theta identity one of his proofs of the functional equation of the zeta function, which we recall here in its symmetrical form:

$$
\pi^{-s / 2} \Gamma\left(\frac{s}{2}\right) \zeta(s)=\pi^{-(1-s) / 2} \Gamma\left(\frac{1-s}{2}\right) \zeta(1-s)
$$

But there is more to be said on the Riemann sums $\sum_{m \in \mathbb{Z}} \frac{1}{|u|} \phi\left(\frac{m}{u}\right)$ from the point of view of their connections with the Fourier Transform than just the Poisson summation formula (2); there holds the co-Poisson intertwining formula ("dualized Poisson formula" of Duffin-Weinberger [13]), which 
reads:

(4)

$$
\mathcal{F}\left(\sum_{m \in \mathbb{Z}, m \neq 0} \frac{g(m / u)}{|u|}-\int_{\mathbb{R}} g(y) d y\right)(t)=\sum_{n \in \mathbb{Z}, n \neq 0} \frac{g(t / n)}{|n|}-\int_{\mathbb{R}} \frac{g(1 / x)}{|x|} d x
$$

We show in [7] that it is enough to suppose for its validity that the integrals $\int_{\mathbb{R}} \frac{g(1 / x)}{|x|} d x$ and $\int_{\mathbb{R}} g(y) d y$ are absolutely convergent. The co-Poisson formula then computes the Fourier Transform of a locally integrable function which is also tempered as a distribution, the Fourier transform having the meaning given to it by Schwartz's theory of tempered distributions. In the case when $g(x)$ is smooth, compactly supported away from $x=0$, then the identity is an identity of Schwartz functions. It is a funny thing that the easiest manner to prove for such a $g(x)$ that the sides of (4) belong to the Schwartz class is to use the Poisson formula (2) itself. So the Poisson formula helps us in understanding the co-Poisson sums, and the co-Poisson formula tells us things on the Poisson-sums.

A most interesting case arises when the function $g(x)$ is an integrable function, compactly supported away from $x=0$, which turns out to have the property that the co-Poisson formula is an identity in $L^{2}(\mathbb{R})$. The author has no definite opinion on whether it is, or is not, an obvious problem to decide which $g(x)$ (compactly supported away from $x=0$ ) will be such that (one, hence) the two sides of the co-Poisson identity are squareintegrable. The only thing one can say so far is that $g(x)$ has to be itself square-integrable.

Note 2. Both the Poisson summation formulae (1), (2), and the co-Poisson intertwining formula (4) tell us $0=0$ when applied to odd functions (taking derivatives leads to further identities which apply non-trivially to oddfunctions.) In all the following we deal only with even functions on the real line. The square integrable among them will be assigned squared-norm $\int_{0}^{\infty}|f(t)|^{2} d t$. We let $K=L^{2}(0, \infty ; d t)$, and we let $\mathcal{F}_{+}$be the cosine transform on $K$ :

$$
\mathcal{F}_{+}(f)(u)=2 \int_{0}^{\infty} \cos (2 \pi t u) f(t) d t
$$

The elements of $K$ are also tacitly viewed as even functions on $\mathbb{R}$.

Let us return to how the functional equation (3) relates with (2) and (4). The left-hand-side of $(3)$ is, for $\operatorname{Re}(s)>1, \int_{0}^{\infty} \sum_{n \geq 1} 2 e^{-\pi n^{2} t^{2}} t^{s-1} d x$. An expression which is valid in the critical strip is:

$$
0<\operatorname{Re}(s)<1 \Rightarrow \pi^{-s / 2} \Gamma\left(\frac{s}{2}\right) \zeta(s)=\int_{0}^{\infty}\left(\sum_{n \geq 1} 2 e^{-\pi n^{2} t^{2}}-\frac{1}{t}\right) t^{s-1} d t
$$


More generally we have the Müntz Formula [24, II.11]:

(5) $0<\operatorname{Re}(s)<1 \Rightarrow$

$$
\zeta(s) \int_{0}^{\infty} \phi(t) t^{s-1} d t=\int_{0}^{\infty}\left(\sum_{n \geq 1} \phi(n t)-\frac{\int_{0}^{\infty} \phi(y) d y}{t}\right) t^{s-1} d t
$$

We call the expression inside the parentheses the modified Poisson sum (so the summation is accompanied with the substracted integral). Replacing $\phi(t)$ with $g(1 / t) /|t|$, with $g(t)$ smooth, compactly supported away from $t=0$, gives a formula involving a co-Poisson sum:

(6) $0<\operatorname{Re}(s)<1 \Rightarrow$

$$
\zeta(s) \int_{0}^{\infty} g(t) t^{-s} d t=\int_{0}^{\infty}\left(\sum_{n \geq 1} \frac{g(t / n)}{n}-\int_{0}^{\infty} \frac{g(1 / y)}{y} d y\right) t^{-s} d t
$$

Let us now write $\widehat{f}(s)=\int_{0}^{\infty} f(t) t^{-s} d t$ for the right Mellin Transform, as opposed to the left Mellin Transform $\int_{0}^{\infty} f(t) t^{s-1} d t$. These transforms are unitary identifications of $K=L^{2}(0, \infty ; d t)$ with $L^{2}\left(s=\frac{1}{2}+i \tau ; d \tau / 2 \pi\right)$. Let $I$ be the unitary operator $I(f)(t)=f(1 / t) /|t|$. The composite $\mathcal{F}_{+} \cdot I$ is scale invariant hence diagonalized by the Mellin Transform, and this gives, on the critical line:

$$
\widehat{\mathcal{F}_{+}(f)}(s)=\chi(s) \widehat{f}(1-s)
$$

with a certain function $\chi(s)$ which we obtain easily from the choice $f(t)=$ $2 \exp \left(-\pi t^{2}\right)$ to be $\pi^{s-1 / 2} \Gamma\left(\frac{1-s}{2}\right) / \Gamma\left(\frac{s}{2}\right)$, hence also $\chi(s)=\zeta(s) / \zeta(1-s)$.

The co-Poisson formula (4) follows then from the functional equation in the form

$$
\zeta(s)=\chi(s) \zeta(1-s)
$$

together with (7) and (6). And the Poisson formula (2) similarly follows from (8) together with (5). We refer the reader to [7] for further discussion and perspectives.

The general idea of the equivalence between the Poisson summation formula (2) and the functional equation (3), with an involvement of the left Mellin Transform $\int_{0}^{\infty} f(t) t^{s-1} d t$, has been familiar and popular for many decades. Recognizing that the right Mellin Transform $\int_{0}^{\infty} f(t) t^{-s} d t$ allows for a distinct Fourier-theoretic interpretation of the functional equation emerged only recently with our analysis [7] of the co-Poisson formula. 


\section{Sonine spaces of de Branges and co-Poisson subspaces}

Let us now discuss some specific aspects of the co-Poisson formula (4) (for an even function):

$$
\mathcal{F}_{+}\left(\sum_{m \geq 1} \frac{g(m / t)}{|t|}-\widehat{g}(0)\right)=\sum_{n \geq 1} \frac{g(t / n)}{n}-\widehat{g}(1)
$$

We are using the right Mellin transform $\widehat{g}(s)=\int_{0}^{\infty} g(t) t^{-s} d t$. Let us take the (even) integrable function $g(t)$ to be with its support in $[a, A]$ (and, as will be omitted from now on, also $[-A,-a]$ of course), with $0<a<A$. Let us assume that the co-Poisson sum $F(t)$ given by the right hand side belongs to $K=L^{2}(0, \infty ; d t)$. It has the property of being equal to the constant $-\widehat{g}(1)$ in $(0, a)$ and with its Fourier (cosine) transform again constant in $(0,1 / A)$. After rescaling, we may always arrange that $a A=1$, which we will assume henceforth, so that $1 / A=a$ (hence, here, $0<a<1$ ).

So we are led to associate to each $a>0$ the sub-Hilbert space $L_{a}$ of $K$ consisting of functions which are constant in $(0, a)$ and with their cosine transform again constant in $(0, a)$. Elementary arguments (such as the ones used in [7, Prop. 6.6]), prove that the $L_{a}$ 's for $0<a<\infty$ compose a strictly decreasing chain of non-trivial infinite dimensional subspaces of $K$ with $K=\overline{\cup_{a>0} L_{a}},\{0\}=\cap_{a>0} L_{a}, L_{a}=\overline{\cup_{b>a} L_{b}}$ (one may also show that $\cup_{b>a} L_{b}$, while dense in $L_{a}$, is a proper subspace). This filtration is a slight variant on the filtration of $K$ which is given by the Sonine spaces $K_{a}, a>0$, defined and studied by de Branges in [2]. The Sonine space $K_{a}$ consists of the functions in $K$ which are vanishing identically, as well as their Fourier (cosine) transforms, in $(0, a)$. The terminology "Sonine spaces", from [22] and [3], includes spaces related to the Fourier sine transform, and also to the Hankel transforms, and is used to refer to some isometric spaces of analytic functions; we will also call $K_{a}$ and $L_{a}$ "Sonine spaces". In the present paper we use only the Fourier cosine tranform.

Theorem 2.1 (De Branges [2]). Let $0<a<\infty$. Let $f(t)$ belong to $K_{a}$. Then its completed right Mellin transform $M(f)(s)=\pi^{-s / 2} \Gamma\left(\frac{s}{2}\right) \widehat{f}(s)$ is an entire function. The evaluations at complex numbers $w \in \mathbb{C}$ are continuous linear forms on $K_{a}$.

We gave an elementary proof of this statement in [6]. See also [8, Théorème 1] for a useful extension. Some slight change of variable is necessary to recover the original de Branges formulation, as he ascribes to the real axis the rôle played here by the critical line. The point of view in [2] is to start with a direct characterization of the entire functions $M(f)(s)$. Indeed a fascinating discovery of de Branges is that the space of functions $M(f)(s), f \in K_{a}$ satisfies all axioms of his general theory of Hilbert spaces 
of entire functions [3] (we use the critical line where [3] always has the real axis). It appears to be useful not to focus exclusively on entire functions, and to allow poles, perhaps only finitely many.

Proposition 2.2 ([7, 6.10]). Let $f(t)$ belong to $L_{a}$. Then its completed right Mellin transform $M(f)(s)=\pi^{-s / 2} \Gamma\left(\frac{s}{2}\right) \widehat{f}(s)$ is a meromorphic function in the entire complex plane, with at most poles at 0 and at 1 . The evaluations $f \mapsto M(f)^{(k)}(w)$ for $w \neq 0, w \neq 1$, or $f \mapsto \operatorname{Res}_{s=0}(M(f))$, $f \mapsto \operatorname{Res}_{s=1}(M(f))$ are continuous linear forms on $L_{a}$. One has the functional equations $M\left(\mathcal{F}_{+}(f)\right)(s)=M(f)(1-s)$.

We will write $Y_{w, k}^{a}$ for the vector in $L_{a}$ with

$$
\forall f \in L_{a} \quad \int_{0}^{\infty} f(t) Y_{w, k}^{a}(t) d t=M(f)^{(k)}(w)
$$

This is for $w \neq 0,1$. For $w=0$ we have $Y_{0}^{a}$ which computes the residue at 0 , and similarly $Y_{1}^{a}$ for the residue at 1 . We are using the bilinear forms $[f, g]=\int_{0}^{\infty} f(t) g(t) d t$ and not the Hermitian scalar product $(f, g)=$ $\int_{0}^{\infty} f(t) \overline{g(t)} d t$ in order to ensure that the dependency of $Y_{w, k}^{a}$ with respect to $w$ is analytic and not anti-analytic. There are also evaluators $Z_{w, k}^{a}$ in the subspace $K_{a}$, which are (for $w \neq 0,1$ ) orthogonal projections from $L_{a}$ to $K_{a}$ of the evaluators $Y_{w, k}^{a}$.

Definition 3. We let $Y_{a} \subset L_{a}$ be the closed subspace of $L_{a}$ which is spanned by the vectors $Y_{\rho, k}^{a}, 0 \leq k<m_{\rho}$, associated to the non-trivial zeros $\rho$ of the Riemann zeta function with multiplicity $m_{\rho}$.

Definition 4. We let the "co-Poisson subspace" $P_{a} \subset L_{a}$, for $0<a<1$, be the subspace of square-integrable functions $F(t)$ which are co-Poisson sums of a function $g \in L^{1}(a, A ; d t)(A=1 / a)$.

The subspace of $K_{a}$ defined analogously to $Y_{a}$ is denoted $Z_{a}$ (rather $Z_{\lambda}$ ) in [7]. The subspace of $K_{a}$ analogous to the co-Poisson subspace $P_{a}$ of $L_{a}$ is denoted $W_{a}^{\prime}$ (rather $W_{\lambda}^{\prime}$ ) in [7]. One has $W_{a}^{\prime}=P_{a} \cap K_{a}$. It may be shown that if the integrable function $g(t)$, compactly supported away from $t=0$, has its co-Poisson sum in $L_{a}$, then $g$ is supported in $[a, A]$ and is square-integrable itself.

\section{Statements of Completeness and Minimality}

It is a non-trivial fact that $P_{a}$ (and $W_{a}^{\prime}$ also, as is proven in [7]) is closed. This is part of the following two theorems.

Theorem 3.1. The vectors $Y_{\rho, k}^{a}, 0 \leq k<m_{\rho}$, associated with the nontrivial zeros of the Riemann zeta function, are a minimal system in $L_{a}$ if and only if $a \leq 1$. They are a complete system if and only if $a \geq 1$. For 
$a<1$ the perpendicular complement to $Y_{a}$ is the co-Poisson subspace $P_{a}$. For $a>1$ we may omit arbitrarily (finitely) many of the $Y_{\rho, k}^{a}$ 's and still have a complete system in $L_{a}$.

Theorem 3.2. The vectors $Z_{\rho, k}^{a}, 0 \leq k<m_{\rho}$, are a minimal, but not complete, system for $a<1$. They are not minimal for $a=1$, but the system obtained from omitting 2 arbitrarily chosen among them (with the convention that one either omits $Z_{\rho, m_{\rho}-1}^{1}$ and $Z_{\rho, m_{\rho}-2}^{1}$ or $Z_{\rho, m_{\rho}-1}^{1}$ and $Z_{\rho^{\prime}, m_{\rho^{\prime}-1}}^{1}$ ) is again a minimal system, which is also complete in $K_{1}$. In the case $a>1$ the vectors $Z_{\rho, k}^{a}$ are complete in $K_{a}$, even after omitting arbitrarily (finitely) many among them.

Remark 5. This is to be contrasted with the fact that the evaluators $Z_{\rho, k}^{1 / \sqrt{q}}$ associated with the non-trivial zeros of a Dirichlet $L$-function $L(s, \chi)$ (for an even primitive character of conductor $q$ ) are a complete and minimal system in $K_{1 / \sqrt{q}}$. Completeness was proven in [7,6.30], and minimality is established as we will do here for the Riemann zeta function.

We use the terminology that an indexed collection of vectors $\left(u_{\alpha}\right)$ in a Hilbert space $K$ is said to be minimal if no $u_{\alpha}$ is in the closure of the linear span of the $u_{\beta}$ 's, $\beta \neq \alpha$, and is said to be complete if the linear span of the $u_{\alpha}$ 's is dense in $K$. To each minimal and complete system is associated a uniquely determined dual system $\left(v_{\alpha}\right)$ with $\left(v_{\beta}, u_{\alpha}\right)=\delta_{\beta \alpha}$ (actually in our $L_{a}$ 's, we use rather the bilinear form $\left.[f, g]=\int_{0}^{\infty} f(t) g(t) d t\right)$. Such a dual system is necessarily minimal, but by no means necessarily complete in general (as an example, one may take $u_{n}=1-z^{n}, n \geq 1$, in the Hardy space of the unit disc. Then $v_{m}=-z^{m}$, for $m \geq 1$, and they are not complete).

For simplicity sake, let us assume that the zeros are all simple. Then, once we know that $\zeta(s) /(s-\rho)$, for $\rho$ a non-trivial zero, belongs to the space $\widehat{L_{1}}$ of (right) Mellin transforms of elements of $L_{1}$, we then identify the system dual to the $Y_{\rho, 0}^{1}$ 's, as consisting of (the inverse Mellin transforms of) the functions $\zeta(s) /\left((s-\rho) \zeta^{\prime}(\rho) \pi^{-\rho / 2} \Gamma(\rho / 2)\right)$. Without any simplifying assumption, we still have that the dual system is obtained from suitable linear combinations (it does not seem very useful to spell them out explicitely) of the functions $\zeta(s) /(s-\rho)^{l}, 1 \leq l \leq m_{\rho}, \rho$ a non-trivial zero.

The proofs of 3.1 and 3.2 are a further application of the technique of [7, Chap.6], which uses a Theorem of Krein on Nevanlinna functions [19, 17]. Another technique is needed to establish the completeness in $\widehat{L_{1}}$ of the functions $\zeta(s) /(s-\rho)^{l}, 1 \leq l \leq m_{\rho}$ :

Theorem 3.3. The functions $\zeta(s) /(s-\rho)^{l}$, for $\rho$ a non-trivial zero and $1 \leq l \leq m_{\rho}$ belong to $\widehat{L_{1}}$. They are minimal and complete in $\widehat{L_{1}}$. The dual 
system consists of vectors given for each $\rho$ by triangular linear combinations of the evaluators $Y_{\rho, k}^{1}, 0 \leq k<m_{\rho}$.

There appears in the proof of 3.3 some computations of residues which are reminiscent of a theorem of Ramanujan which is mentioned in Titchmarsh [24, IX.8.].

The last section of the paper deals with the zeros of an arbitrary Sonine functions, and with the properties of the associated evaluators. We obtain in particular a density result on the distribution of its zeros, with the help of the powerful tools from the classical theory of entire functions [20].

\section{Aspects of Sonine functions}

Note 6. We let $\widehat{L_{a}}$ be the vector space of right Mellin transforms of elements of $L_{a}$ (and similarly for $\widehat{K_{a}}$ ). They are square-integrable functions on the critical line, which, as we know from 2.2 are also meromorphic in the entire complex plane. We are not using here the Gamma-completed Mellin transform, but the bare Mellin transform $\widehat{f}(s)$, which according to 2.2 has trivial zeros at $-2 n, n>0$, and possibly a pole at $s=1$, and possibly does not vanish at $s=0$.

Definition 7. We let $\mathbb{H}^{2}$ be the Hardy space of the right half-plane $\operatorname{Re}(s)>$ $\frac{1}{2}$. We simultaneously view $\mathbb{H}^{2}$ as a subspace of $L^{2}\left(\operatorname{Re}(s)=\frac{1}{2},|d s| / 2 \pi\right)$ and as a space of analytic functions in the right half-plane. We also use selfexplanatory notations such as $A^{s} \mathbb{H}^{2}$.

The right Mellin transform is an isometric identification of $L^{2}(1, \infty ; d t)$ with $\mathbb{H}^{2}$ : this is one of the famous theorems of Paley-Wiener [21], after a change of variable. Hence, for $0<a$ and $A=1 / a$, the right Mellin transform is an isometric identification of $L^{2}(a, \infty ; d t)$ with $A^{s} \mathbb{H}^{2}$. Furthermore, the right Mellin transform is an isometric identification of $\mathbb{C} \cdot \mathbf{1}_{0<t<a}+L^{2}(a, \infty ; d t)$ with $\frac{s}{s-1} A^{s} \mathbb{H}^{2}$. This leads to the following characterization of $\widehat{L_{a}}$ :

Proposition 4.1. The subspace $\widehat{L_{a}}$ of $L^{2}\left(\operatorname{Re}(s)=\frac{1}{2},|d s| / 2 \pi\right)$ consists of the measurable functions $F(s)$ on the critical line which belong to $\frac{s}{s-1} A^{s} \mathbb{H}^{2}$ and are such that $\chi(s) F(1-s)$ also belongs to $\frac{s}{s-1} A^{s} \mathbb{H}^{2}$. Such a function $F(s)$ is the restriction to the critical line of an analytic function, meromorphic in the entire complex plane with at most a pole at $s=1$, and with trivial zeros at $s=-2 n, n \in \mathbb{N}, n>0$.

Proof. We know already from 2.2 that functions in $\widehat{L_{a}}$ have the stated properties. If a function $F(s)$ belongs to $\frac{s}{s-1} A^{s} \mathbb{H}^{2}$, viewed as a space of (equivalence classes of) measurable functions on the critical line, then it is square-integrable and is the Mellin transform of an element $f(t)$ of 
$\mathbb{C} \cdot \mathbf{1}_{0<t<a}+L^{2}(a, \infty ; d t)$. We know that the Fourier cosine transform of $f$ has $\chi(s) F(1-s)$ as Mellin transform, so the second condition on $F$ tells us that $f$ belongs to $L_{a}$.

We recall that $\chi(s)$ is the function (expressible in terms of the Gamma function) which is involved in the functional equation of the Riemann zeta function (8), and is in fact the spectral multiplier of the scale invariant operator $\mathcal{F}_{+} \cdot I$, for the right Mellin transform.

Note 8. Abusively, we will say that $\chi(s) F(1-s)$ is the Fourier transform of $F(s)$, and will sometimes even write $\mathcal{F}_{+}(F)(s)$ instead of $\chi(s) F(1-s)$. It is useful to take note that if we write $F(s)=\zeta(s) \theta(s)$ we then have $\chi(s) F(1-s)=\zeta(s) \theta(1-s)$.

Proposition 4.2. The functions $\zeta(s) /(s-\rho)^{l}, 1 \leq l \leq m_{\rho}$ associated with the non-trivial zeros of the Riemann zeta function belong to $\widehat{L_{1}}$.

Proof. The function $F(s)=\zeta(s) /(s-\rho)^{l}$ is square-integrable on the critical line. And $\chi(s) F(1-s)=(-1)^{l} \zeta(s) /(s-(1-\rho))^{l}$. So we only need to prove that $\frac{s-1}{s} F(s)=\frac{s-1}{s} \zeta(s) /(s-\rho)^{l}$ belongs to $\mathbb{H}^{2}$. This is well-known to be true of $\frac{s-1}{s} \zeta(s) / s$ (from the formula $\zeta(s) / s=1 /(s-1)-\int_{1}^{\infty} \frac{\{t\}}{t} t^{-s} d t$, valid for $0<\operatorname{Re}(s))$, hence it holds also for $\frac{s-1}{s} \zeta(s) / s^{l}$. If we exclude a neigborhood of $\rho$ then $s^{l} /(s-\rho)^{l}$ is bounded, so going back to the definition of $\mathbb{H}^{2}$ as a space of analytic functions in the right half-plane with a uniform bound of their $L^{2}$ norms on vertical lines we obtain the desired conclusion.

The following will be useful later:

Proposition 4.3. If $G(s)$ belongs to $\widehat{L_{a}}$ and $s(s-1) \pi^{-s / 2} \Gamma\left(\frac{s}{2}\right) G(s)$ vanishes at $s=w$ then $G(s) /(s-w)$ again belongs to $\widehat{L_{a}}$. If $G(s)$ belongs to $\widehat{K_{a}}$ and $\pi^{-s / 2} \Gamma\left(\frac{s}{2}\right) G(s)$ vanishes at $s=w$ then $G(s) /(s-w)$ again belongs to $\widehat{K_{a}}$.

Proof. We could prove this in the "t-picture", but will do it in the " $s$ picture". We see as in the preceding proof that $G(s) /(s-w)$ still belongs to $\frac{s}{s-1} A^{s} \mathbb{H}^{2}$. The entire function $s(s-1) \pi^{-s / 2} \Gamma\left(\frac{s}{2}\right) G(s)$ vanishes at $s=w$ so $s(s-1) \pi^{-s / 2} \Gamma\left(\frac{s}{2}\right) \mathcal{F}_{+}(G)(s)$ vanishes at $s=1-w$ and the same argument then shows that $\chi(s) G(1-s) /(1-s-w)$ belongs to $\frac{s}{s-1} A^{s} \mathbb{H}^{2}$. We then apply Proposition 4.1. The statement for $K_{a}$ is proven analogously.

Note 9. It is a general truth in all de Branges' spaces that such a statement holds for zeros $w$ off the symmetry axis (which is here the critical line). This is, in fact, almost one of the axioms for de Branges' spaces. The possibility to divide by $(s-w)$ if $w$ is on the symmetry axis depends on whether the structure function $E$ (on this, we refer to [3]) is not vanishing or vanishing 
at $w$. For the Sonine spaces, the proposition 4.3 proves that the structure functions $E_{a}(z)$ have no zeros on the symmetry axis. For more on the $E_{a}(z)$ 's and allied functions, see [8] and [9].

A variant on this gives:

Lemma 4.4. If $F(s)$ belongs to $\widehat{K_{a}}$ then $F(s) / s$ belongs to $\widehat{L_{a}}$.

Proof. The function $F(s) / s$ (which is regular at $s=0$ ) belongs to the space $A^{s} \mathbb{H}^{2}$, simply from $1 /|s|=O(1)$ on $\operatorname{Re}(s) \geq \frac{1}{2}$. Its image under the Fourier transform is $\mathcal{F}_{+}(F)(s) /(1-s)$ which belongs to $\frac{s}{s-1} A^{s} \mathbb{H}^{2}$.

Proposition 4.5. One has $\operatorname{dim}\left(L_{a} / K_{a}\right)=2$.

Proof. This is equivalent to the fact that the residue-evaluators $Y_{0}^{a}$ and $Y_{1}^{a}$ are linearly independent in $L_{a}$, which may be established in a number of elementary ways; we give two proofs. Evaluators off the symmetry axis are always non-trivial in de Branges spaces so there is $F(s) \in \widehat{K_{a}}$ with $F^{\prime}(0) \neq 0$ (one knows further From [6, Théorème 2.3.] that any finite system of vectors $Z_{w, k}^{a}$ in $K_{a}$ is a linearly independent system). So we have $F(s) / s=G(s) \in \widehat{L_{a}}$ not vanishing at 0 but with no pole at 1 . Its "Fourier transform" $\chi(s) G(1-s)$ vanishes at 0 but has a pole at 1 . This proves $\operatorname{dim}\left(L_{a} / K_{a}\right) \geq 2$ and the reverse equality follows from the fact that the subspace $K_{a}$ is defined by two linear conditions.

For the second proof we go back to the argument of [6] which identifies the perpendicular complement to $K_{a}$ in $L^{2}(0, \infty ; d t)$ to be the closed space $L^{2}(0, a)+\mathcal{F}_{+}\left(L^{2}(0, a)\right)$. It is clear that $L_{a}$ is the perpendicular complement to the (two dimensions) smaller space $\left(L^{2}(0, a) \cap \mathbf{1}_{0<t<a}^{\perp}\right)+\mathcal{F}_{+}\left(L^{2}(0, a) \cap\right.$ $\left.\mathbf{1}_{0<t<a}^{\perp}\right)$ and this proves 4.5 .

The technique of the second proof has the additional benefit:

Proposition 4.6. The union $\bigcup_{b>a} K_{b}$ is dense in $K_{a}$, and $\bigcup_{b>a} L_{b}$ is dense in $L_{a}$.

Proof. Generally speaking $\bigcap_{b>a}\left(A_{b}+B_{b}\right)=\left(\cap_{b>a} A_{b}\right)+\left(\cap_{b>a} B_{b}\right)$ when we have vector spaces indexed by $b>a$ with $A_{b_{1}} \subset A_{b_{2}}$ and $B_{b_{1}} \subset B_{b_{2}}$ for $b_{1}<b_{2}$ and $A_{b} \cap B_{b}=\{0\}$ for $b>a$. We apply this to $A_{b}=L^{2}(0, b ; d t)$ and $B_{b}=\mathcal{F}_{+}\left(L^{2}(0, b ; d t)\right)$, as $K_{a}=\left(A_{a}+B_{a}\right)^{\perp}, A_{a}=\cap_{b>a} A_{b}, B_{a}=\cap_{b>a} B_{b}$, and $A_{a}+B_{a}$ is closed as a subspace of $L^{2}(0, \infty ; d t)$.

Proposition 4.7. The vector space $\bigcup_{b>a} K_{b}$ is properly included in $K_{a}$ and the same holds for the respective subspaces of Fourier invariant, or skew, functions (and similarly for $L_{a}$ ).

Proof. Let $g \in K_{b}$, with $b>a$ and $g$ having the leftmost point of its support at $b$. Then $g(b t / a)$ has the leftmost point of its support at $a$. If $g$ 
is invariant under Fourier then we use $\sqrt{\frac{b}{a}} g(b t / a)+\sqrt{\frac{a}{b}} g(a t / b)$ to obtain again an invariant function, with leftmost point of its support at $a$.

Definition 10. We say that a function $F(s)$, analytic in $\mathbb{C}$ with at most finitely many poles, has the L-Property if the estimates $F(\sigma+i \tau)=$ $O_{a, b, \epsilon}\left((1+|\tau|)^{\left(\frac{1}{2}-a\right)^{+}+\epsilon}\right)$ hold (away from the poles), for $-\infty<a \leq \sigma \leq$ $b<\infty, \epsilon>0$.

Theorem 4.8. The functions in $\widehat{L_{a}}$ have the L-Property.

Proof. Let $g(t)$ be a function in $L_{a}$ and let $G(s)=\int_{0}^{\infty} g(t) t^{-s} d t$ be its right Mellin transform. The function $g(t)$ is a constant $\alpha(g)$ on $(0, a)$. An expression for $G(s)$ as a meromorphic function (in $\frac{1}{2}<\operatorname{Re}(s)<1$, hence) in the right half-plane is:

$$
\begin{aligned}
G(s)= & \frac{-\alpha(g) a^{1-s}}{s-1}+\int_{a}^{\infty} g(t) t^{-s} d t \\
= & \frac{-\alpha(g) a^{1-s}}{s-1}+\int_{0}^{\infty} \mathcal{F}_{+}\left(\mathbf{1}_{t>a} t^{-s}\right)(u) \mathcal{F}_{+}(g)(u) d u \\
= & \frac{-\alpha(g) a^{1-s}}{s-1}+\alpha\left(\mathcal{F}_{+}(g)\right) \int_{0}^{a} \mathcal{F}_{+}\left(\mathbf{1}_{t>a} t^{-s}\right)(u) d u \\
& +\int_{a}^{\infty} \mathcal{F}_{+}\left(\mathbf{1}_{t>a} t^{-s}\right)(u) \mathcal{F}_{+}(g)(u) d u
\end{aligned}
$$

We established in [6] a few results of an elementary nature about the functions $\mathcal{F}_{+}\left(\mathbf{1}_{t>a} t^{-s}\right)(u)$ which are denoted there $C_{a}(u, 1-s)$ (in particular we showed that these functions are entire functions of $s)$. For $\operatorname{Re}(s)<1$ one has according to $[6$, eq. 1.3.]:

$$
\mathcal{F}_{+}\left(\mathbf{1}_{t>a} t^{-s}\right)(u)=\chi(s) u^{s-1}-2 \sum_{j=0}^{\infty} \frac{(-1)^{j}}{(2 j) !}(2 \pi u)^{2 j} \frac{a^{2 j+1-s}}{2 j+1-s}
$$

hence for $0<\operatorname{Re}(s)<1$ :

$$
\int_{0}^{a} \mathcal{F}_{+}\left(\mathbf{1}_{t>a} t^{-s}\right)(u) d u=\frac{\chi(s) a^{s}}{s}-2 \sum_{j=0}^{\infty} \frac{(-1)^{j}(2 \pi)^{2 j}}{(2 j) !} \frac{a^{4 j+2-s}}{(2 j+1)(2 j+1-s)}
$$

This is bounded on $\frac{1}{4} \leq \operatorname{Re}(s) \leq \frac{3}{4}$ (using the well-known uniform estimate $|\chi(s)| \sim|\operatorname{Im}(s) / 2 \pi|^{-\operatorname{Re}(s)+1 / 2}$ as $|\operatorname{Im}(s)| \rightarrow \infty$ in vertical strips [24, IV.12.3.]). We also have from integration by parts and analytic continuation to $\operatorname{Re}(s)>0$ the expression:

$$
\mathcal{F}_{+}\left(\mathbf{1}_{t>a} t^{-s}\right)(u)=\frac{s \int_{a}^{\infty} \sin (2 \pi u t) t^{-s-1} d t-a^{-s} \sin (2 \pi u a)}{\pi u}
$$


which is $O(|s| / u)$ on $\frac{1}{4} \leq \operatorname{Re}(s) \leq \frac{3}{4}, 0<u$. Combining all this we find the estimate:

$$
G(s)=O(|s|) \text { on } \frac{1}{4} \leq \operatorname{Re}(s) \leq \frac{3}{4}
$$

This (temporary) estimate justifies the use of the Phragmén-Lindelöf principle from bounds on $\operatorname{Re}(s)=\frac{1}{2} \pm \epsilon$. On any half-plane $\operatorname{Re}(s) \geq \frac{1}{2}+\epsilon>\frac{1}{2}$ (excluding of course a neighborhood of $s=1$ ) one has $G(s)=O\left(A^{\operatorname{Re}(s)}\right)$ from the fact that $(s-1) G(s) / s$ belongs to $A^{s} \mathbb{H}^{2}$ and that elements of $\mathbb{H}^{2}$ are bounded in $\operatorname{Re}(s) \geq \frac{1}{2}+\epsilon>\frac{1}{2}$. And the functional equation $G(1-s)=\chi(1-s) \widehat{\mathcal{F}_{+}(g)}(s)$ gives us estimates on the left half-plane. This shows that the L-Property holds for $G(s)$. In particular, the Lindelöf exponents $\mu_{G}(\sigma)$ are at most 0 for $\sigma \geq \frac{1}{2}$ and at most $\frac{1}{2}-\sigma$ for $\sigma \leq \frac{1}{2}$.

Remark 11. In fact, the proof given above establishes the L-Property for $G(s)$ in a stronger form than stated in the definition 10. One has for example $G(s)=O_{\eta}\left((1+|\operatorname{Im}(s)|)^{\eta}\right)$ for each $\eta>0$, on the strip $\frac{1}{2}-\eta \leq$ $\operatorname{Re}(s) \leq \frac{1}{2}, G(s)=O_{\epsilon}\left(|s|^{\epsilon}\right)$ for each $\epsilon>0$ on $\frac{1}{2} \leq \operatorname{Re}(s) \leq 1$ (away from the allowed pole at $s=1)$, and $G(s)=O_{\eta}\left(A^{\operatorname{Re}(s)}\right)$ on $\operatorname{Re}(s) \geq \frac{1}{2}+\eta, \eta>0$.

Definition 12. We let $\mathcal{L}_{1}$ to be the sub-vector space of $L_{1}$ containing the functions $g(t)$ whose right-Mellin transforms $G(s)$ are $O_{g, a, b, N}\left(|s|^{-N}\right)$ on all vertical strips $a \leq \operatorname{Re}(s) \leq b$, and for all integers $N \geq 1$ (away from the pole, and the implied constant depending on $g, a, b$, and $N$ ).

Theorem 4.9. The sub-vector space $\mathcal{L}_{1}$ is dense in $L_{1}$.

Proof. From proposition 4.6 we only have to show that any function $G(s)$ in a $\widehat{L_{b}}, b>1$ is in the closure of $\widehat{\mathcal{L}_{1}}$. For this let $\theta(s)$ be the Mellin transform of a smooth function with support in $[1 / e, e]$, satisfying $\theta\left(\frac{1}{2}\right)=1$. The function $\theta(s)$ is an entire function which decreases faster than any (inverse) power of $|s|$ as $|\operatorname{Im}(s)| \rightarrow \infty$ in any given strip $a \leq \sigma \leq b$. Let us consider the functions $G_{\epsilon}(s)=\theta\left(\epsilon\left(s-\frac{1}{2}\right)+\frac{1}{2}\right) G(s)$ as $\epsilon \rightarrow 0$. On the critical line they are dominated by a constant multiple of $|G(s)|$ so they are square-integrable and converge in $L^{2}$-norm to $G(s)$. We prove that for $1 \leq \exp (-\epsilon) b<b$ these functions all belong to $\mathcal{L}_{1}$. Their quick decrease in vertical strips is guaranteed by the fact that $G(s)$ has the L-Property. The function

$$
\frac{s-1}{s} G_{\epsilon}(s)=\theta\left(\epsilon\left(s-\frac{1}{2}\right)+\frac{1}{2}\right) \frac{s-1}{s} G(s)
$$

on the critical line is the Mellin transform of a multiplicative convolution on $(0, \infty)$ of an element in $L^{2}(b, \infty)$ with a smooth function supported in $[\exp (-\epsilon), \exp (+\epsilon)]$. The support of this multiplicative convolution will be included in $[1, \infty)$ if $1 \leq \exp (-\epsilon) b$. So for those $\epsilon>0$ one has $G_{\epsilon}(s) \epsilon$ $\frac{s}{s-1} \mathbb{H}^{2}$. Its image under $\mathcal{F}_{+}$is $\theta\left(-\epsilon\left(s-\frac{1}{2}\right)+\frac{1}{2}\right) \mathcal{F}_{+}(G)(s)=\theta^{\tau}\left(\epsilon\left(s-\frac{1}{2}\right)+\right.$ $\left.\frac{1}{2}\right) \mathcal{F}_{+}(G)(s)$ where $\theta^{\tau}(w)=\theta(1-w)$ has the same properties as $\theta(w)$ (we 
recall our abusive notation $\mathcal{F}_{+}(F)(s)=\chi(s) F(1-s)$.) Hence $\mathcal{F}_{+}\left(G_{\epsilon}\right)(s)$ also belongs to $\frac{s}{s-1} \mathbb{H}^{2}$ and this completes the proof that $G_{\epsilon}(s) \in \widehat{\mathcal{L}_{1}}$.

Lemma 4.10. The subspace $\mathcal{L}_{1}$ is stable under $\mathcal{F}_{+}$.

Proof. Clear from the estimates of $\chi(s)$ in vertical strips ([24, IV.12.3.]).

\section{Completeness of the system of functions $\zeta(s) /(s-\rho)$}

We will use a classical estimate on the size of $\zeta(s)^{-1}$ :

Proposition 5.1 (from [24, IX.7.]). There is a real number $A$ and a strictly increasing sequence $T_{n}>n$ such that $|\zeta(s)|^{-1}<|s|^{A}$ on $|\operatorname{Im}(s)|=T_{n}$, $-1 \leq \operatorname{Re}(s) \leq+2$.

Note 13. From now on an infinite sum $\sum_{\rho} a(\rho)$ (with complex numbers or functions or Hilbert space vectors a $(\rho)$ 's indexed by the non-trivial zeros of the Riemann zeta function) means $\lim _{n \rightarrow \infty} \sum_{|\operatorname{Im}(\rho)|<T_{n}} a(\rho)$, where the limit might be, if we are dealing with functions, a pointwise almost everywhere limit, or a Hilbert space limit. When we say that the partial sums are bounded (as complex numbers, or as Hilbert space vectors) we only refer to the partial sums as written above. When we say that the series is absolutely convergent it means that we group together the contributions of the $\rho$ 's with $T_{n}<|\operatorname{Im}(\rho)|<T_{n+1}$ before evaluating the absolute value or Hilbert norm. When building series of residues we write sometimes things as if the zeros were all simple: this is just to make the notation easier, but no hypothesis is made in this paper on the multiplicities $m_{\rho}$, and the formula used for writing $a(\rho)$ is a symbolic representation, valid for a simple zero, of the more complicated expression which would apply in case of multiplicity, which we do not spell out explicitely.

Theorem 5.2. Let $G(s)$ be a function in $\widehat{L_{1}}$ which belongs to the dense subspace $\widehat{\mathcal{L}_{1}}$ of functions with quick decrease in vertical strips. Then the series of residues for a fixed $Z \neq 1$, not a zero:

$$
\sum_{\rho} \frac{G(\rho)}{\zeta^{\prime}(\rho)} \frac{\zeta(Z)}{Z-\rho}
$$

converges absolutely pointwise to $G(Z)$ on $\mathbb{C} \backslash\{1\}$. It also converges absolutely in $L^{2}$-norm to $G(Z)$ on the critical line.

This is a series of residues for $\frac{G(s)}{\zeta(s)} \frac{\zeta(Z)}{Z-s}$ where $s$ is the variable and $Z \neq 1$ is a parameter (with the exception of the residue at $s=Z$ ). We have written the contribution of $\rho$ as if it was simple (Titchmarsh uses a simlar convention in [24, IX.8.]). In fact the exact expression is a linear combination of $\zeta(Z) /(Z-\rho)^{l}, 1 \leq l \leq m_{\rho}$. We note that the trivial zeros and $s=1$ are not singularities and contribute no residue. 
Proof of Theorem 5.2. Let us consider first the pointwise convergence. We fix $Z$, not 1 and not a zero and consider the function of $s$

$$
\frac{G(s)}{\zeta(s)} \frac{\zeta(Z)}{Z-s}
$$

We apply the calculus of residues to the contour integral around a rectangle with corners $\frac{1}{2} \pm A \pm i T_{n}$ where $A \geq \frac{3}{2}$ is chosen sufficiently large such that both $Z$ and $1-Z$ are in the open rectangle when $n$ is large enough. Thanks to 5.1 and the fact that $G(s)$ has quick decrease the contribution of the horizontal segments vanish as $n \rightarrow \infty$. The contribution of the vertical segments converge to the (Lebesgue convergent) integral over the vertical lines and we obtain:

$$
\sum_{\rho} \frac{G(\rho)}{\zeta^{\prime}(\rho)} \frac{\zeta(Z)}{Z-\rho}-G(Z)=\frac{1}{2 \pi}\left(\int_{\operatorname{Re}(s)=\frac{1}{2}+A}-\int_{\operatorname{Re}(s)=\frac{1}{2}-A}\right) \frac{\zeta(Z) G(s)}{(Z-s) \zeta(s)}|d s|
$$

We prove that the vertical contributions vanish. The functional equation

$$
\frac{G(1-s)}{\zeta(1-s)}=\frac{\mathcal{F}_{+}(G)(s)}{\zeta(s)}
$$

reduces the case $\operatorname{Re}(s)=\frac{1}{2}-A$ to the case $\operatorname{Re}(s)=\frac{1}{2}+A$. That last integral does not change when we increase $A$. We note that the $L^{2}$-norms of $G(s)$ on $\operatorname{Re}(s)=\sigma \geq 2$ are uniformly bounded because this is true with $G(s)$ replaced with $(s-1) G(s) / s$ (which belongs to a Hardy space). Also $1 / \zeta(s)=O(1)$ in $\operatorname{Re}(s) \geq 2$. The Cauchy-Schwarz inequality then shows that the integral goes to 0 as $A \rightarrow \infty$. This proves the pointwise convergence:

$$
G(Z)=\sum_{\rho} \frac{G(\rho)}{\zeta^{\prime}(\rho)} \frac{\zeta(Z)}{Z-\rho}
$$

Going back to the contribution of the zeros with $T_{n}<|\operatorname{Im}(\rho)|<T_{n+1}$, and expressing it as a contour integral we see using $G(s) \in \mathcal{L}_{1}$ and Proposition 5.1 that the series of residues is clearly absolutely convergent (with the meaning explained in Note 13).

To show that the series converges to $G(Z)$ in $L^{2}$ on the critical line it will be enough to prove it to be absolutely convergent in $L^{2}$. We may with the same kind of reasoning prove the absolute convergence of the series of residues:

$$
\sum_{\rho} \frac{G(\rho)}{\zeta^{\prime}(\rho)}
$$

For this we consider $G(s) / \zeta(s)$ along rectangles with vertical borders on $\operatorname{Re}(s)=\frac{1}{2} \pm \frac{3}{2}$ and horizontal borders at the $\pm T_{n}$ and $\pm T_{n+1}$. The functional equation (to go from $\operatorname{Re}(s)=-1$ to $\operatorname{Re}(s)=2$ ), the estimate 5.1 and the fact that $G(s)$ belongs to $\mathcal{L}_{1}$ then combine to prove that this series of 
residues is absolutely convergent. In fact it converges to 0 as we prove later, but this is not needed here. So returning to the problem of $L^{2}$-convergence we need only prove the $L^{2}$ absolute convergence on the critical line of:

$$
\begin{aligned}
& \sum_{\rho} \frac{G(\rho)}{\zeta^{\prime}(\rho)}\left(\frac{\zeta(Z)}{Z-\rho}-\frac{\zeta(Z)}{Z+2}\right) \\
= & \sum_{\rho} \frac{G(\rho)}{\zeta^{\prime}(\rho)} \zeta(Z) \frac{2+\rho}{(Z-\rho)(Z+2)}
\end{aligned}
$$

And for this it will be sufficient to prove the $L^{2}$ absolute convergence of:

$$
\sum_{\rho} \frac{G(\rho)}{\zeta^{\prime}(\rho)} \zeta(Z) \frac{Z-1}{Z+2} \frac{2+\rho}{(Z-\rho)(Z+2)}
$$

We note that the function $(Z-1) \zeta(Z) /(Z+2)^{3}$ belongs to $\mathbb{H}^{2}\left(\operatorname{Re}(s) \geq-\frac{1}{2}\right)$, hence the same holds for each of the function above depending on $\rho$. In case of a multiple zero its contribution must be re-interpreted as a residue and will be as a function of $Z$ a linear combination of the $(Z-1) \zeta(Z) /(Z-$ $\rho)^{l}(Z+2)^{2}, 1 \leq l \leq m_{\rho}$, which also belong to $\mathbb{H}^{2}\left(\operatorname{Re}(s) \geq-\frac{1}{2}\right)$. It will thus be enough to prove that the series above is $L^{2}$-absolutely convergent on the line $\operatorname{Re}(Z)=-\frac{1}{2}$, as the norms are bigger on this line than on the critical line. We may then remove one factor $(Z-1) /(Z+2)$ and we are reduced to show that

$$
\sum_{\rho} \frac{G(\rho)}{\zeta^{\prime}(\rho)} \frac{\zeta(Z)(2+\rho)}{(Z-\rho)(Z+2)}
$$

is $L^{2}$-absolutely convergent on $\operatorname{Re}(Z)=-\frac{1}{2}$. What we do now is to reexpress for each $Z$ on this line the contributions of the zeros with $T_{n}<$ $|\operatorname{Im}(\rho)|<T_{n+1}$ as a contour integral on the rectangles (one with positive imaginary parts and the other its reflection in the horizontal axis) bordered vertically by $\operatorname{Re}(s)=-\frac{1}{4}$ and $\operatorname{Re}(s)=+\frac{5}{4}$. This will involve along this contour the function of $s$ :

$$
\frac{G(s)}{\zeta(s)} \frac{\zeta(Z)(2+s)}{(Z-s)(Z+2)}
$$

For a given fixed $s$ with $-\frac{1}{4} \leq \operatorname{Re}(s) \leq+\frac{5}{4}$ the function of $Z$ on $\operatorname{Re}(Z)=-\frac{1}{2}$ given by

$$
\frac{\zeta(Z)(2+s)}{(Z+2)(Z-s)}
$$

has its $L^{2}$ norm which is $O\left(1+|s|^{2}\right)$. Indeed:

$$
\frac{2+s}{Z-s}=\frac{2+s}{Z} \frac{Z}{Z-s}=\frac{2+s}{Z}\left(1+\frac{s}{Z-s}\right)=\frac{O\left(1+|s|^{2}\right)}{|Z|}
$$


and $\zeta(Z) / Z(Z+2)$ is square-integrable on $\operatorname{Re}(Z)=-\frac{1}{2}$. The integrals along these rectangular contours of the absolute values $\left(1+|s|^{2}\right)|G(s)| /|\zeta(s)|$ give, from the quick decrease of $G(s)$ and the Proposition 5.1, a convergent series. With this the proof of 5.2 is complete.

This gives:

Corollary 5.3. The functions $\zeta(s) /(s-\rho)^{l}, 1 \leq l \leq m_{\rho}$ associated with the non-trivial zeros are a complete system in $\widehat{L_{1}}$.

We also take note of the following:

Proposition 5.4. One has for each $G(s)$ in the dense subspace $\widehat{\mathcal{L}_{1}}$ :

$$
0=\sum_{\rho} \frac{G(\rho)}{\zeta^{\prime}(\rho)}
$$

where the series of residues is absolutely convergent.

Proof. We have indicated in the proof of 5.2 that the series is absolutely convergent and its value is

$$
\frac{1}{2 \pi}\left(\int_{\sigma=2}-\int_{\sigma=-1}\right) \frac{G(s)}{\zeta(s)}|d s|
$$

We prove that the $\sigma=2$ integral vanishes, and the $\sigma=-1$ integral will then too also from the functional equation

$$
\frac{G(1-s)}{\zeta(1-s)}=\frac{\mathcal{F}_{+}(G)(s)}{\zeta(s)}
$$

where $\mathcal{F}_{+}(G)$ also belongs to $\widehat{\mathcal{L}_{1}}$. Using on $\sigma=2$ the absolutely convergent expression $\frac{1}{\zeta(s)}=\sum_{k \geq 1} \mu(k) k^{-s}$ it will be enough to prove:

$$
0=\frac{1}{2 \pi} \int_{\sigma=2} G(s) k^{-s}|d s|
$$

We shift the integral to the critical line and obtain

$$
\frac{1}{2 \pi} \int_{\sigma=\frac{1}{2}} G(s) k^{-s}|d s|+\frac{\operatorname{Res}_{1}(G)}{k}
$$

On the critical line we have in the $L^{2}$-sense $G(s)=\int_{0}^{\infty} f(t) t^{s-1} d t$ for a certain square-integrable function $f(t)$ (which is $g(1 / t) / t$ with $G(s)=\widehat{g}(s)$ ). The Fourier-Mellin inversion formula gives, in square-mean sense:

$$
f(t)=\lim _{T \rightarrow+\infty} \frac{1}{2 \pi} \int_{s=\frac{1}{2}-i T}^{s=\frac{1}{2}+i T} G(s) t^{-s}|d s|
$$

As $G(s)$ is $O\left(|s|^{-N}\right)$ on the critical line for arbitrary $N$, we find that $f(t)$ is a smooth function on $(0, \infty)$ given pointwise by the above formula. From 
the definition of $L_{1}$ one has $f(t)=c / t$ for $t \geq 1$ with a certain constant c. The function $\int_{0}^{1} f(t) t^{s-1} d t$ is analytic for $\operatorname{Re}(s)>\frac{1}{2}$ so the residue of $G(s)$ comes from $\int_{1}^{\infty} c \cdot t^{s-2} d t$. This is first for $\operatorname{Re}(s)<1$ then by analytic continuation the function $-c /(s-1)$ so $\operatorname{Res}_{1}(G)=-c$, and on the other hand $f(k)=+c / k$. Combining all this information the proof is complete.

Remark 14. The result is (slightly) surprising at first as we will prove that the evaluators associated with the zeros are a complete and minimal system.

Remark 15. These computations of residues are reminiscent of a formula of Ramanujan which is mentioned in Titchmarsh [24, IX.8.]. For $a b=$ $\pi, a>0$ :

$$
\sqrt{a} \sum_{n=1}^{\infty} \frac{\mu(n)}{n} e^{-(a / n)^{2}}-\sqrt{b} \sum_{n=1}^{\infty} \frac{\mu(n)}{n} e^{-(b / n)^{2}}=-\frac{1}{2 \sqrt{b}} \sum_{\rho} b^{\rho} \frac{\Gamma\left(\frac{1-\rho}{2}\right)}{\zeta^{\prime}(\rho)}
$$

where the meaning of the sum over the zeros is the one from Note 13.

\section{Completion of the proofs of $3.1,3.2,3.3$}

We also prove that the evaluators associated with the zeros are a complete system in $L_{1}$. This is a further application of the technique of [7, Chap. 6] which uses the theory of Nevanlinna functions and especially that part of a fundamental theorem of Krein [19] which says that an entire function which is Nevanlinna in two complementary half-planes is necessarily of finite exponential type (see e.g. [17, I.§4]).

Proposition 6.1. Let $a \geq 1$. The vectors $Y_{\rho, k}^{a}$ associated with the nontrivial zeros of the Riemann zeta function are complete in $L_{a}$.

Proof. If $g \in L_{a}$ is perpendicular to all those vectors (hence also $\mathcal{F}_{+}(g)$ ) then its right Mellin transform $G(s)$ factorizes as:

$$
G(s)=\zeta(s) \theta(s)
$$

with an entire function $\theta(s)$. We have used that $G(s)$ shares with $\zeta(s)$ its trivial zeros and has at most a pole of order 1 at $s=1$. This expression proves that $\theta(s)$ belongs to the Nevanlinna class of the right half-plane (as $G(s)$ and $\zeta(s)$ are meromorphic functions in this class). From the functional equation:

$$
\theta(1-s)=\frac{G(1-s)}{\zeta(1-s)}=\frac{\widehat{\mathcal{F}_{+}(g)}(s)}{\zeta(s)}
$$

we see that $\theta(s)$ also belongs to the Nevanlinna class of the left half-plane. According to the theorem of Krein $[19,17]$ it is of finite exponential type 
which (if $\theta$ is not the zero function) is given by the formula:

$$
\max \left(\limsup _{\sigma \rightarrow+\infty} \frac{\log |\theta(\sigma)|}{\sigma}, \limsup _{\sigma \rightarrow+\infty} \frac{\log |\theta(1-\sigma)|}{\sigma}\right)
$$

We know that $G(s) / \zeta(s)$ is $O\left(A^{\operatorname{Re}(s)}\right.$ ) (with $A=1 / a$ ) in $\operatorname{Re}(s) \geq 2$ and similarly for $\mathcal{F}_{+}(G)(s) / \zeta(s)$. So this settles the matter for $A<1(a>1)$ as the formula gives a strictly negative result. For $a=1$ we obtain that $\theta(s)$ is of minimal exponential type. From the expression $G(s) / \zeta(s)$ on $\operatorname{Re}(s)=2$ it is square-integrable on this line. From the Paley-Wiener Theorem [21] being of minimal exponential type it in fact vanishes identically.

Proposition 6.2. Let $a>1$. The vectors $Y_{\rho, k}^{a}$ associated with the nontrivial zeros of the Riemann zeta function are not minimal: indeed they remain a complete system in $L_{1}$ even after omitting arbitrarily finitely many among them.

Proof. We adapt the proof of the preceding proposition to omitting the vectors associated with the zeros from a finite set $R$. The starting point will be

$$
G(s)=\frac{\zeta(s)}{\prod_{\rho \in R}(s-\rho)^{m_{\rho}}} \theta(s)
$$

for a certain entire function $\theta(s)$. The Krein formula for its exponential type again gives a strictly negative result. So $\theta$ vanishes identically.

Theorem 6.3. Let $a=1$. The vectors $Y_{\rho, k}^{1}$ associated with the non-trivial zeros of the Riemann zeta function are a minimal (and complete) system in $L_{1}$. The vectors, inverse Mellin transforms of the functions $\zeta(s) /(s-\rho)^{l}$, $1 \leq l \leq m_{\rho}$, are a minimal (and complete) system in $L_{1}$.

Proof. The fact that the functions $\zeta(s) /(s-\rho)^{l}, 1 \leq l \leq m_{\rho}$ belong to $\widehat{L_{1}}$ implies that the evaluators $Y_{\rho, k}^{1}$ 's are a minimal system. We know already that they are a complete system. The system of the $\zeta(s) /(s-\rho)^{l}, 1 \leq$ $l \leq m_{\rho}$, is, up to triangular invertible linear combinations for each $\rho$ the uniquely determined dual system. As a dual system it has to be minimal. And we know already from 5.3 that it is a complete system.

This completes the proof of 3.3 .

Proposition 6.4. Let $a<1$. The vectors $Y_{\rho, k}^{a}$ are minimal and not complete in $L_{a}$.

Proof. If they were not minimal, their orthogonal projections to $L_{1}$ which are the vectors $Y_{\rho, k}^{1}$, would not be either. And they are not complete from the existence of the co-Poisson subspace $P_{a}$. 
With this the proof of 3.1 is completed, with the exception of the identification of the co-Poisson space as the perpendicular complement to the space spanned by the $Y_{\rho, k}^{a}$ 's. We refer the reader to [7, Chap.6] especially to $[7$, Theorems $6.24,6.25]$ which have all the elements for the proof, as it does not appear useful to devote space to this here.

Proposition 6.5. The vectors $Z_{\rho, k}^{1}$ are not minimal in $K_{1}$. In fact $K_{1}$ is spanned by these vectors even after omitting $Z_{\rho_{1}, m_{\rho_{1}}-1}^{1}$ and $Z_{\rho_{2}, m_{\rho_{2}}-1}^{1}$ $\left(\rho_{1} \neq \rho_{2}\right)$, or $Z_{\rho, m_{\rho}-1}^{1}$ and $Z_{\rho, m_{\rho}-2}^{1}\left(m_{\rho} \geq 2\right)$, from the list. This shortened system is then a minimal system.

Proof. If $f$ in $K_{1}$ is perpendicular (for the form $[f, g]=\int_{1}^{\infty} f(t) g(t) d t$ ) to this shortened list of evaluators then its right Mellin transform factorizes as

$$
F(s)=\frac{s(s-1) \zeta(s)}{\left(s-\rho_{1}\right)\left(s-\rho_{2}\right)} \theta(s)
$$

where we have used that $F(0)=0$ and that $F(s)$ has no pole at $s=1$. In this expression we have the two cases $\rho_{1} \neq \rho_{2}$ and $\rho_{1}=\rho_{2}$. The proof then proceeds as above and leads to $F(s)=0$. To prove minimality for the shortened system one only has to consider the functions

$$
\frac{s(s-1) \zeta(s)}{\left(s-\rho_{1}\right)\left(s-\rho_{2}\right)} \frac{1}{(s-\rho)^{l}}
$$

associated with the remaining zeros (and remaining multiplicities), as they are easily seen to be the right Mellin transforms of elements from the Sonine space $K_{1}$.

Proposition 6.6. Let $a>1$. The vectors $Z_{\rho, k}^{a}$ span $K_{a}$ even after omitting arbitrarily finitely many among them.

Proof. They are the orthogonal projections to $K_{a}$ of the vectors $Y_{\rho, k}^{a}$ in $L_{a}$.

Theorem 6.7. Let $a<1$. The vectors $Z_{\rho, k}^{a}$ are minimal in $K_{a}$.

Proof. Let $\theta(t)$ be a smooth non-zero function supported in $[a, A](A=$ $1 / a>1)$. Its right Mellin transform $\widehat{\theta}(s)$ is then $O\left(A^{|\operatorname{Re}(s)|}\right)$ on $\mathbb{C}$. And if $P(s)$ is an arbitrary polynomial, then $P(s) \widehat{\theta}(s)=\widehat{\theta_{P}}(s)$ for a certain smooth function $\theta_{P}$, again supported in $[a, A]$, so $\widehat{\theta_{P}}(s)=O_{P}\left(A^{|\operatorname{Re}(s)|}\right)$. Hence $\widehat{\theta}(s)$ decreases faster than any inverse polynomial in any given vertical strip, in particular on $-1 \leq \operatorname{Re}(s) \leq 2$. From this we see that the function $G(s)=s(s-1) \widehat{\theta}(s) \zeta(s)$ is square-integrable on the critical line and belongs to $A^{s} \mathbb{H}^{2}$ (one may write $G(s)=s^{3} \widehat{\theta}(s)(s-1) \zeta(s) / s^{2}$, and use the fact that $(s-1) \zeta(s) / s^{2}$ belongs to $\left.\mathbb{H}^{2}\right)$. We have $\chi(s) G(1-s)=s(s-1) \widehat{\theta}(1-s) \zeta(s)$ so again this belongs to $A^{s} \mathbb{H}^{2}$. This means that $G(s)$ is the right Mellin 
transform of a (non-zero) element $g$ of $K_{a}$. Let us now take a non-trivial zero $\rho$, which for simplicity we assume simple. We choose the function $\theta(t)$ to be such that $\widehat{\theta}(\rho) \neq 0$, which obviously may always be arranged. Then, using $4.2, G(s) /(s-\rho)$ is again the Mellin transform of a non-zero element $g_{\rho}$ in $K_{a}$. This element is perpendicular (for the bilinear form $[f, g]$ ) to all the evaluators except $Z_{\rho, 0}^{a}$, to which it is not perpendicular. So $Z_{\rho, 0}^{a}$ can not be in the closed span of the others. The proof is easily extended to the case of a multiple zero (we don't do this here, as the next section contains a proof of a more general statement).

The three theorems 3.1, 3.2, 3.3 are thus established.

\section{Zeros and evaluators for general Sonine functions}

Let us more generally associate to any non-empty multiset $\mathcal{Z}$ of complex numbers (a countable collection of complex numbers, each assigned a finite multiplicity) the problem of determining whether the associated evaluators are minimal, or complete in a Sonine space $K_{a}$ or an extended Sonine space $L_{a}$. To be specific we consider the situation in $K_{a}$, the discussion could be easily adapted to $L_{a}$. From the fact that the Sonine spaces are a decreasing chain, with evaluators in $K_{a}$ projecting orthogonally to the evaluators in $K_{b}$ for $b \geq a$, we may associate in $[0,+\infty]$ two indices $a_{1}(\mathcal{Z})$ and $a_{2}(\mathcal{Z})$ to the multiset $\mathcal{Z} \in \mathbb{C}$. The index $a_{1}(\mathcal{Z})$ will be such that the evaluators are a minimal system for $a<a_{1}(\mathcal{Z})$ and not a minimal system for $a>a_{1}(\mathcal{Z})$ and the index $a_{2}(\mathcal{Z})$ will be such that the evaluators are complete for $a>a_{2}(\mathcal{Z})$ but not complete for $a<a_{2}(\mathcal{Z})$. Let us take for example the multiset $\mathcal{Z}$ to have an accumulation point $w$ (there is for each $\epsilon>0$ at least one complex number $z$ in the support of $\mathcal{Z}$ with $0<|z-w|<\epsilon)$ : then the system is never minimal and is always complete so that $a_{1}=0$ and $a_{2}=0$. As another example we take the multiset to have finite cardinality: then the evaluators are always minimal and never complete so $a_{1}=+\infty$, and $a_{2}=+\infty$. For the zeros of the Riemann zeta function we have $a_{1}=a_{2}=1$. There is a general phenomenon here:

Theorem 7.1. The equality $a_{1}(\mathcal{Z})=a_{2}(\mathcal{Z})$ always holds.

Let us thus write $a(\mathcal{Z})$ for either $a_{1}(\mathcal{Z})$ or $a_{2}(\mathcal{Z})$. We will prove that $a(\mathcal{Z})$ does not change from adding or removing a finite multiset to $\mathcal{Z}$ (maintaining $\mathcal{Z}$ non-empty):

Theorem 7.2. If $0<a<a(\mathcal{Z})$ then the evaluators associated to $\mathcal{Z}$ remain not complete, and minimal, in $K_{a}$, after including arbitrarily finitely many other evaluators.

Theorem 7.3. If $a(\mathcal{Z})<a<\infty$ then the evaluators associated to $\mathcal{Z}$ remain complete, and not minimal, in $K_{a}$ after omitting arbitrarily finitely many among them. 
We will say that $g(t)$ is a Sonine function if it belongs to $\cup_{a>0} K_{a} \subset$ $L^{2}(0, \infty ; d t)$. We also say that $G(s)$ is a Sonine function if it is the right Mellin transform of such a $g(t)$.

Lemma 7.4. If the system of evaluators associated in a given $K_{a}$ to a (non-empty) multiset $\mathcal{Z}$ is not complete, then it is minimal. Alternatively, if it is not minimal, it has to be complete.

Proof. Let us assume that the system is not complete. Then we have a non zero Sonine function $G(s)$ in $\widehat{K_{a}}$ such that $\pi^{-s / 2} \Gamma\left(\frac{s}{2}\right) G(s)$ vanishes on $\mathcal{Z}$. From the proposition 4.3 we know that if $\pi^{-w / 2} \Gamma\left(\frac{w}{2}\right) G(w)=0$ then $G(s) /(s-w)$ is again a Sonine function in $\widehat{K_{a}}$. Let us now proceed to take $\rho$ in the support of $\mathcal{Z}$, and divide $G(s)$ by powers of $(s-\rho)$ to construct functions which vanish exactly to the $k$-th order at $\rho$, for $0 \leq k<$ $m_{\mathcal{Z}}(\rho)$ (this is after incorporating the Gamma factor). From suitable linear combinations we construct further an $a$-Sonine function $G_{k}(s)$ whose $l$-th derivative for $0 \leq l<m_{\mathcal{Z}}(\rho)$ (again with the Gamma factor incorporated) vanishes at $\rho$, except for $l=k$ for which it does not vanish, and with $G_{k}(s)$ vanishing on the remaining part of the multiset $\mathcal{Z}$. This proves that the evaluators in $K_{a}$ associated with $\mathcal{Z}$ are minimal.

We note that this provides an alternative route to our statement from [6] that finitely many evaluators are always linearly independent in $K_{a}$, once we know that $K_{a}$ is infinite dimensional.

Lemma 7.5. If the system of evaluators associated in a given $K_{a}$ to a (non-empty) multiset $\mathcal{Z}$ is minimal, then it is not complete in any $K_{b}$ with $b<a$.

Proof. We pick a $\rho$ in the support of $\mathcal{Z}$, with multiplicity $m_{\rho}$. As the system is minimal, we have the existence of at least one Sonine function $G(s)$ in $\widehat{K_{a}}$ which vanishes on the other part of $\mathcal{Z}$ but vanishes only to the $\left(m_{\rho}-1\right)$-th order at $\rho$. Let us now consider a function $F(s)=\theta(s) G(s)$ where $\theta(s)$ is the Mellin transform of a non-zero smooth function supported in an interval $[\exp (-\epsilon), \exp (+\epsilon)]$. We know from Theorem 4.8 that Sonine functions have the L-Property, so using the arguments of the smoothing technique in the proof of Theorem 4.9 we obtain easily that any such $F(s)$ is a non-zero element of $\widehat{L_{b}}$ for any $b \leq \exp (-\epsilon) a$. Replacing $\theta(s)$ by $(s-\rho) \theta(s)$ we may impose $\theta(\rho)=0$. Then $F(s)$ (with the Gamma factor) vanishes on $\mathcal{Z}$ and this proves that the evaluators associated with $\mathcal{Z}$ are not complete in $\widehat{L_{b}}$.

At this stage we have completed the proof of Theorem 7.1: Lemma 7.4 implies $a_{2}(\mathcal{Z}) \leq a_{1}(\mathcal{Z})$ and Lemma 7.5 implies $a_{1}(\mathcal{Z}) \leq a_{2}(\mathcal{Z})$. 
Lemma 7.6. If the system of evaluators associated in a given $K_{a}$ to a (non-empty) multiset $\mathcal{Z}$ is minimal, then it is not complete in any $K_{b}$ with $b<a$, even after adding to the system of evaluators associated with $\mathcal{Z}$ arbitrarily finitely many other evaluators.

Proof. We only have to replace the function $\theta(s)$ from the preceding proof by $P(s) \theta(s)$ where $P(s)$ is an arbitrary polynomial.

This, together with Lemma 7.4, clearly implies Theorem 7.2. It also implies the Theorem 7.3: let us suppose $a(\mathcal{Z})<a<\infty$. Let us imagine that after removing finitely many evaluators we do not have a complete system. Then this remaining system, being not complete, has to be minimal from Lemma 7.4. We just proved that in these circumstances the system in a $K_{b}$ with $b<a$ can not be complete, even after including finitely many arbitrary evaluators. This gives a contradiction for $a(\mathcal{Z})<b<a$, as we may reintegrate the omitted evaluators. So Theorem 7.3 holds.

Let $g$ be a non-zero Sonine function. We write $\lambda(g)>0$ for the minimal point of the support of $g$ and $\mu(g)>0$ for the minimal point of the support of $\mathcal{F}_{+}(g)$. And we let $a(g)$ be $\sqrt{\lambda(g) \mu(g)}$.

To each non-zero Sonine function $g$ we associate the multiset $\mathcal{Z}_{g}$ (which will be proven to have infinite cardinality) of its non-trivial zeros: these are the zeros of the completed Mellin transform $\pi^{-s / 2} \Gamma\left(\frac{s}{2}\right) \widehat{g}(s)$, so $0,-2, \ldots$, might be among them but they are counted with multiplicity one less than in $\widehat{g}(s)$.

Before proceeding further we need to recall some classical results from the Theory of Nevanlinna functions and Hardy Spaces. We refer the reader for example to [3, Chap.1] and [17, I.§4] for proofs and more detailed statements (see also $[15,16,18]$ ). A Nevanlinna function $F(s)$ in a half-plane (we consider here $\left.\operatorname{Re}(s)>\frac{1}{2}\right)$ is an analytic function which may be written as the quotient of two bounded analytic functions. To each non-zero $F$ is associated a real-number $h(F)$, its mean type (in the terminology from [3]), which may be obtained (in the case of the half-plane $\operatorname{Re}(s)>\frac{1}{2}$ ) from the formula $h(F)=\lim \sup _{\sigma \rightarrow+\infty} \log |F(\sigma)| / \sigma$. The mean-type of a product is the sum of the mean types. The mean-type contributes a factor $e^{h\left(s-\frac{1}{2}\right)}$ to the Nevanlinna-Smirnov factorization of the function $F(s)$, in particular, for the specific case of the Smirnov-Beurling factorization of an element in $\mathbb{H}^{2}$, it gives the special inner factor (here $h \leq 0$ ). The other factors have mean type 0 . In the particular case when $F(s)$ is the right Mellin transform of a square-integrable function $f(t)$ supported in $[\lambda,+\infty), \lambda>0$, then the mean-type of $F$ is also $\log \left(\lambda(f)^{-1}\right)$ where $\lambda(f) \geq \lambda>0$ is the lowest point of the support of $f$. To see this, we may after a multiplicative translation assume that $\lambda(f)=1$. We want to prove that the mean-type of $F$ is 0 . One has $h \leq 0$ as $F$ is bounded, say for $\operatorname{Re}(s) \geq 1$. In the canonical 
factorization of $F$, the outer factor is still an element of the Hardy space. The inner factor is bounded by 1 . So $F(s)$ belongs to $e^{h s} \mathbb{H}^{2}$, which is the subspace of Mellin transforms of $L^{2}\left(e^{-h}, \infty ; d t\right)$, so $h=0$. We conclude this brief summary with Krein's theorem [19], which we have already used in the previous proofs. This important theorem (see [17, I.§4]) states in particular that an entire function $\theta(s)$ which is in the Nevanlinna class in two complementary half-planes is necessarily of finite exponential type. Furthermore the exponential type is the maximum of the mean-types for the two half-planes. Hence, if $\theta$ is not the zero function, at least one of the two mean-types has to be non-negative.

We will also need some classical results from the theory of entire functions [20]. Let $F(z)$ be an entire function. Then $F$ is said $([20, \mathrm{I} . \S 12])$ to have normal type with respect to the (Lindelöf) refined (proximate) order $r \log (r)$ (which is the one useful to us here) if

$$
0<\limsup _{r \rightarrow \infty} \frac{\log \max _{|z|=r}|F(z)|}{r \log (r)}<\infty
$$

If this holds, the generalized Phragmén-Lindelöf indicator function is defined as:

$$
h_{F}(\theta)=\limsup _{r \rightarrow \infty} \frac{\log \left|F\left(r e^{i \theta}\right)\right|}{r \log (r)}
$$

One proves that the indicator function of the entire function $F(z)$ of normal type is finite valued and is a continuous "trigonometrically convex" function of $\theta([20, \mathrm{I} . \S 18])$.

Remark 16. The indicator function for $F(z-a)$ is the same as the one for $F(z)$ : to see this one may use the upper estimate [20, I.§18, Thm 28] $\log \left|F\left(r e^{i \vartheta}\right)\right|<\left(h_{F}(\vartheta)+\epsilon\right) r \log (r)$ for $r>r_{\epsilon}$ in a given open angular sector $|\arg (z)-\theta|<\eta$, and the continuity of $h_{F}$ at $\theta$. The parallel ray starting at $a$ is contained in this sector except for a finite segment, so the indicator function based at $a$ is bounded above by the one based at the origin, and vice versa.

A ray $L_{\theta}=\left\{r e^{i \theta}, 0<r<\infty\right\}$ is a ray of completely regular growth (CRG-ray, [20, III]) for $F$ if

$$
h_{F}(\theta)=\lim _{r \rightarrow \infty, r \notin E_{\theta}} \frac{\log \left|F\left(r e^{i \theta}\right)\right|}{r \log (r)}
$$

where the excluded set $E_{\theta} \subset(0, \infty)$ has vanishing upper relative linear density. The set of CRG-rays is closed. The entire function $F(z)$ is said to be of completely regular growth if all the rays are CRG-rays. A fundamental theorem [20, III. $\S 3]$ which applies to CRG-functions states that the number $n(r, \alpha, \beta)$ of zeros of modulus at most $r$ in the open angular sector $\alpha<\theta<\beta$ 
has the following asymptotic behavior:

$$
\lim _{r \rightarrow \infty} \frac{n(r, \alpha, \beta)}{r \log (r)}=\frac{1}{2 \pi}\left(h_{F}^{\prime}(\beta)-h_{F}^{\prime}(\alpha)+\int_{\alpha}^{\beta} h_{F}(\theta) d \theta\right)
$$

under the condition that $h_{F}$ admits derivatives at $\alpha$ and $\beta$ (from the trigonometrical convexity right and left derivatives always exist).

Theorem 7.7. Let $g(t)$ be a non-zero Sonine function, with Mellin transform $G(s)$, and Gamma-completed Mellin transform $\mathcal{G}(s)$. The entire function $\mathcal{G}(s)$ is of normal type for the Lindelöf refined order $r \log (r)$. Its indicator function is $\frac{1}{2}|\cos (\theta)|$. The entire function $\mathcal{G}(s)$ is a function of completely regular growth. The number of its zeros of modulus at most $T$ in the angular sector $\left|\arg \left(z-\frac{1}{2}\right)-\frac{\pi}{2}\right|<\epsilon<\pi$ is asymptotically equivalent to $\frac{T}{2 \pi} \log (T)$, and similarly for the angular sectors containing the lower-half of the critical line. The number of zeros of $\mathcal{G}(s)$ with modulus at most $T$ in $|\arg ( \pm z)|<\frac{\pi}{2}-\epsilon$ is $o(T)$.

Proof. We know from Theorem 4.8 that $G(s)$ has the L-property, and in particular it is $O(1+|s|)$ in $0 \leq \operatorname{Re}(s) \leq 1$, and furthermore it is $O\left(A^{\operatorname{Re}(s)}\right)$ in $\operatorname{Re}(s) \geq 1$. On the other hand the Stirling formula easily leads to

$$
\lim _{r \rightarrow \infty} \frac{\max _{\left|s-\frac{1}{2}\right|=r, \operatorname{Re}(s) \geq 0} \log \left|\pi^{-s / 2} \Gamma\left(\frac{s}{2}\right)\right|}{r \log (r)}=\frac{1}{2}
$$

so certainly

$$
\limsup _{r \rightarrow \infty} \frac{\max _{\left|s-\frac{1}{2}\right|=r, \operatorname{Re}(s) \geq 0} \log |\mathcal{G}(s)|}{r \log (r)} \leq \frac{1}{2}
$$

As further $\lim \sup _{\sigma \rightarrow \infty} \log |G(\sigma)| / \sigma$ is finite (it is the mean-type of $G$ in the right-half plane) we have $\lim \sup _{\sigma \rightarrow \infty} \log |G(\sigma)| / \sigma \log (\sigma)=0$. Hence:

$$
\limsup _{r \rightarrow \infty} \frac{\max _{\left|s-\frac{1}{2}\right|=r, \operatorname{Re}(s) \geq 0} \log |\mathcal{G}(s)|}{r \log (r)}=\frac{1}{2}
$$

In $\operatorname{Re}(s) \leq 1$ we have the identical result as $\mathcal{G}(1-s)$ is the completed Mellin transform of $\mathcal{F}_{+}(g)$. So the entire function $\mathcal{G}(s)$ is of normal type $\frac{1}{2}$ for the refined order $r \log (r)$. The argument using the Stirling formula which has led to the inequality above gives on any given ray with $|\arg (\theta)| \leq \frac{\pi}{2}$ that its indicator function is bounded above by $\frac{1}{2} \cos (\theta)$ (we use again that for $|\theta|=\frac{\pi}{2}$ we have $G(s)=O(|s|)$, and for $|\theta|<\frac{\pi}{2}$ we have $\left.G(s)=O_{\theta}\left(A^{\operatorname{Re}(s)}\right)\right)$. We show the property of complete regular growth on a ray with $|\theta|<\frac{\pi}{2}$ and at the same time identify the value of the indicator for this ray to be $\frac{1}{2} \cos (\theta)$ (by continuity we will then have the value of the indicator for $|\theta|=\frac{\pi}{2}$ ). The Gamma factor gives the correct limit and there are no excluded values for $r$; so we only need to show that $\log |G(s)| / r \log (r)$ goes to 0 as $r \rightarrow \infty$ while avoiding an exceptional set $E_{\theta} \subset(0, \infty)$ having vanishing upper relative 
linear density $\left(r=\left|s-\frac{1}{2}\right|\right)$. Actually this holds with $r$ replacing $r \log (r)$ and a finite (not necessarily zero) limit, as $A^{-s} G(s)$ belongs to the Hardy space of the right half-plane (and then we can invoke [20, V.§4, Thm 6]; it is all a matter of understanding the CRG-behavior of a Blaschke product, as the other factors in the canonical factorization are easily taken care of). Going back to $\mathcal{G}(s)$ we thus have its CRG property (for the refined order $r \log (r)$ ) on the rays $\arg \left(s-\frac{1}{2}\right)=\theta,|\theta|<\pi / 2$. Hence also in the left half-plane as $\mathcal{G}(1-s)$ is the completed Mellin transform of $\mathcal{F}_{+}(g)$ and the indicator function is thus $\frac{1}{2}|\cos (\theta)|$. As the set of CRG-rays is closed, we conclude that $\mathcal{G}(s)$ is a CRG entire function. The central result from [20, III] leads then to the stated asymptotic densities of zeros in open sectors containing either the upper half or the lower half of the critical line. Concerning the sectors $|\arg ( \pm z)|<\frac{\pi}{2}-\epsilon$, the vanishing asymptotic linear density of the zeros follows again from $[20, \mathrm{~V} . \S 4$, Thm 6$]$, or more simply from the fact that $\sum \frac{1}{|\rho|}$ converges for the zeros in such a sector (the zeros of the Blaschke product satisfy $\left.\sum \frac{\operatorname{Re}(\rho)-\frac{1}{2}}{|\rho|^{2}}<\infty\right)$.

Remark 17. In particular the function $\mathcal{G}(s)$ is an entire function of order one (and maximal type for this order) which admits a representation as an Hadamard product $s^{N} e^{\alpha+\beta s} \prod_{\rho}(1-s / \rho) e^{s / \rho}$.

Remark 18. The entire function $\mathcal{G}\left(\frac{1}{2}+i z\right)$ is a function of the "class A" as studied in $[20, \mathrm{~V}]$.

Remark 19. In [8] we have produced explicit formulae for some even distributions $A_{a}(t)$ and $B_{a}(t)$ having the Sonine property for the cosine transform. We proved that their complete Mellin transforms $\mathcal{A}_{a}(s)$ and $\mathcal{B}_{a}(s)$ are the structure functions of the de Branges Sonine-cosine spaces (no explicit formula had been known prior to [8]): and this has the interesting corollary that the Riemann Hypothesis holds true for them. We have presented in [9] a summary of further results of ours. The Note contains formulae for some second order differential operators intrinsically associated with the Fourier Transform. Under suitable boundary conditions these operators are self-adjoint with discrete spectrum, and the squared imaginary parts of the zeros of $\mathcal{A}_{a}(s)$ and $\mathcal{B}_{a}(s)$ are their eigenvalues (this proves in another manner that $\mathcal{A}_{a}(s)$ and $\mathcal{B}_{a}(s)$ satisfy the Riemann Hypothesis). If $\rho$ is a fixed chosen zero then $\mathcal{A}_{a}(s) /(s-\rho)$ is the complete Mellin transform of a square-integrable even function with the Sonine property. We may then apply Theorem 7.7 with the result that $\mathcal{A}_{a}(s)$ (or $\mathcal{B}_{a}(s)$ ) share with $\pi^{-s / 2} \Gamma\left(\frac{s}{2}\right) \zeta(s)$ the principal order of its asymptotic density of zeros.

Theorem 7.8. For each non-zero Sonine function there holds: $a\left(\mathcal{Z}_{g}\right)=$ $a(g)$. This means in particular that $g$ has infinitely many (non-trivial) zeros, that the evaluators associated to $\mathcal{Z}_{g}$ are minimal but not complete in 
$K_{a}$ if $a \leq a(g)$ and that they are complete, even after omitting arbitrarily finitely many among them, in $K_{a}$ if $a>a(g)$.

Proof. Let $g(t)$ be a non-zero Sonine function in $K_{a}$, with Mellin transform $G(s)$. Let us consider the multi-set $\mathcal{Z}_{g}$ of the non-trivial zeros of $G(s)$ and the associated evaluators in Sonine spaces $K_{b}$. Replacing $g$ by a multiplicative translate we may arrange that the lowest point of its support coincides with the lowest point of the support of $\mathcal{F}_{+}(g)$, hence with the number we have denoted $a(g)$, so we may assume $a=a(g)$. The system of evaluators in $K_{a}$ is not complete, as all are perpendicular to $g$, hence $a\left(\mathcal{Z}_{g}\right) \geq a$. Let $b>a$ and let us prove that the evaluators are complete in $K_{b}$. If not, there is a non-zero function $f(t)$ in $K_{b}$ such that its Mellin transform $F(s)$ factorizes as $F(s)=G(s) \theta(s)$ with an entire function $\theta(s)$. In particular $\theta(s)$ is a Nevanlinna function in the right half-plane. We know that the mean types are related through $h(F)=h(G)+h(\theta)$. We know that $h(G)=-\log a$ and that $h(F) \leq-\log b$, hence $h(\theta) \leq \log (a / b)<0$. On the other hand we have $\chi(s) F(1-s)=\chi(s) G(1-s) \theta(1-s)$. Repeating the argument for $\theta(1-s)$ we obtain that its mean type is also $<0$. According to Krein's theorem $\theta(s)$ has finite exponential type given by the formula

$$
\max \left(\limsup _{\sigma \rightarrow \infty} \frac{\log |\theta(\sigma)|}{\sigma}, \limsup _{\sigma \rightarrow \infty} \frac{\log |\theta(1-\sigma)|}{\sigma}\right),
$$

hence we obtain a strictly negative result. This is impossible, and the function $f \in K_{b}$ does not exist. So the evaluators associated to $\mathcal{Z}$ are complete in $K_{b}$ for $b>a(g)$ and $a(\mathcal{Z}) \leq a=a(g)$. We know already $a(\mathcal{Z}) \geq a(g)$ so we have an equality, as was to be proven. The other statements are just repetitions of previously proven assertions.

We extract from the proof above the following:

Proposition 7.9. If $f$ and $g$ are two non-zero Sonine functions such that $\mathcal{Z}_{g} \subset \mathcal{Z}_{f}$ then the entire function $F(s) / G(s)$ has finite exponential type and $a(f) \leq a(g)$.

Proof. We replace $g$ by a multiplicative translate so that $g \in K_{a}$ with $a=a(g)$. And we similarly assume $f \in K_{b}$ with $b=a(f)$. Krein's theorem is applied to the entire function $\theta(s)$ with $F(s)=G(s) \theta(s)$, with the conclusion that $\theta(s)$ has finite exponential type. Furthermore exactly as in the previous proof if we had $b>a$ we could prove that the mean types of $\theta(s)$ in the left and right half-plane are both strictly negative, which is impossible. So $b \leq a$, that is $a(f) \leq a(g)$.

We also mention:

Proposition 7.10. If $f$ and $g$ are two non-zero Sonine functions such that $\mathcal{Z}_{f}=\mathcal{Z}_{g}$ then $f$ and $g$ are multiplicative translates of one another, up to multiplication by a non-zero complex number. 
Proof. The Hadamard product representation leads to an equality $\widehat{f}(s)=$ $e^{\lambda+\mu s} \widehat{g}(s)$. But Wiener's theorem [21] on the gain of a causal filter tells us that $\log |\widehat{g}(s)|$ and $\log |\widehat{f}(s)|$ are both integrable against a Cauchy weight on the critical line, and this implies that $\mu$ has to be real. The equation then says exactly that $f$ is, up to a multiplicative constant, a multiplicative translate of $g$.

\section{Conclusion}

Our theorems from [7, Chap. 6] concerning the completeness of the evaluators associated to the Riemann zeta function and the Dirichlet Lfunctions have been shown here to be special instances of a more general statement. Does this mean that these theorems from [7] are not specific enough to tell us anything interesting?

To discuss this, we shall, briefly, mention a few basic aspects of the general theory of Hilbert spaces of entire functions, and thus see why it is reasonable to be hopeful of some connections with the problem of the Riemann hypothesis, at the technical level at least (it is in the exact same manner, no more no less, that the, more widely known, basic aspects of the Hilbert theory of self-adjoint operators may be thought of bearing some relevance to the technical aspects of the Riemann hypothesis). We include this short paragraph despite the prolonged existence of ethically unfortunate claims. To each de Branges space are associated (up to some normalizations) a function $\mathcal{A}(z)$ and also a function $\mathcal{B}(z)$ which both have all their zeros on the symmetry axis. This is a corollary to the way the functions $\mathcal{A}(z)$ and $\mathcal{B}(z)$ are related to the Hilbert space structure, hence participates of the general idea of thinking about the Riemann Hypothesis in Hilbert space and operator-theoretical terms. We mention that the work of de Branges is closely related to the vast investigations of M.G. Krein [17] on problems of extrapolation of stationary processes, problems of scattering theory, problems of moments, canonical systems, ..., where the operator theoretical aspects are quite explicitely in the foreground. The zeta function is not entire, but has only one pole. Its functional equation involves Gamma factors, to which de Branges associates the two-parameter family of the Sonine spaces for the Hankel transforms of parameter $\nu$, and the support conditions of parameter $a$. We focus on the spaces associated with the cosine and sine transforms. De Branges $[4,5]$ uses in his constructions the other Sonine spaces, even "double-Sonine" spaces: the idea of using 2dimensional constructs to study a Riemann Hypothesis in dimension 1 is a familiar one from other contexts. The structure functions $\mathcal{A}_{a}(z)$ and $\mathcal{B}_{a}(z)$, especially for the Sonine spaces associated with the cosine transform, have analytic properties and symmetries quite close to what is known to hold for $\pi^{-s / 2} \Gamma(s / 2) \zeta(s)$, with one interesting bonus: they are proven to satisfy 
the Riemann Hypothesis. Explicit representations for these functions, as completed Mellin transforms, have been obtained recently ([8]). We proved here (7.7) that they have to first order the same density of zeros as the Riemann zeta function. We have obtained ([9]) a spectral interpretation of their zeros, in terms of some Dirac and Schrödinger operators which we have associated to the Fourier Transform.

We explained in [7] the path which has led to our own interest in all this: the path from the explicit formula to the co-Poisson formula and beyond. The co-Poisson formula leads to the association with the zeta function of certain quotient spaces of the Sonine spaces. We saw in the previous section that some of the theorems originally proven for the zeta function or the Dirichlet $L$-functions have more general validity, as some aspects hold true for all Sonine functions. The Riemann Hypothesis of course does not hold for all Sonine functions (we may always add arbitrarily chosen zeros; it is also easy to construct an example of a Sonine function with no zeros in the critical strip). Nevertheless it might be that some other aspects, known or expected to hold for the Riemann zeros, do have some amount of wider validity; further investigations of the zeros of Sonine functions are needed to better understand the situation.

Acknowledgments. I thank Michel Balazard and Éric Saias, for discussion on Sonine spaces, and especially on the functions $\zeta(s) /(s-\rho)$.

\section{References}

[1] R. P. BoAs, Sums representing Fourier transforms, Proc. Am. Math. Soc. 3 (1952), 444447.

[2] L. De Branges, Self-reciprocal functions, J. Math. Anal. Appl. 9 (1964) 433-457.

[3] L. De Branges, Hilbert spaces of entire functions, Prentice Hall Inc., Englewood Cliffs, 1968.

[4] L. De Branges, The convergence of Euler products, J. Funct. Anal. 107 (1992), no. 1, $122-210$.

[5] L. De Branges, A conjecture which implies the Riemann hypothesis, J. Funct. Anal. 121 (1994), no. 1, 117-184.

[6] J.-F. BuRnol, Sur certains espaces de Hilbert de fonctions entières, liés à la transformation de Fourier et aux fonctions L de Dirichlet et de Riemann, C. R. Acad. Sci. Paris, Ser. I 333 (2001), 201-206.

[7] J.-F. Burnol, On Fourier and Zeta(s), 50 p., Habilitationsschrift (2001-2002), Forum Mathematicum, to appear (2004).

[8] J.-F. Bunnol, Sur les "espaces de Sonine" associés par de Branges à la transformation de Fourier, C. R. Acad. Sci. Paris, Ser. I 335 (2002), 689-692.

[9] J.-F. Burnol, Des équations de Dirac et de Schrödinger pour la transformation de Fourier, C. R. Acad. Sci. Paris, Ser. I 336 (2003), 919-924.

[10] R. J. Duffin, Representation of Fourier integrals as sums I, Bull. Am. Math. Soc. 51 (1945), 447-455.

[11] R. J. Duffin, Representation of Fourier integrals as sums II, Proc. Am. Math. Soc. 1 (1950), 250-255. 
[12] R. J. Duffin, Representation of Fourier integrals as sums III, Proc. Am. Math. Soc. 8 (1957), 272-277.

[13] R. J. Duffin, H. F. Weinberger, Dualizing the Poisson summation formula, Proc. Natl. Acad. Sci. USA 88 (1991), 7348-7350.

[14] R. J. Duffin, H. F. Weinberger, On dualizing a multivariable Poisson summation for mula, Journ. of Fourier Anal. and Appl. 3 (5) (1997), 487-497.

[15] H. Dym, H.P. McKean, Fourier series and integrals, Academic Press, 1972.

[16] H. Dym, H.P. McKean, Gaussian processes, function theory, and the inverse spectral problem, Probability and Mathematical Statistics, Vol. 31. Academic Press, New YorkLondon, 1976.

[17] M. L. Gorbachuk, V. I. Gorbachuk, M. G. Krein's lectures on entire operators, Operator Theory: Advances and Applications, 97. Birkhäuser Verlag, Basel, 1997.

[18] K. Hoffman, Banach spaces of analytic functions, Reprint of the 1962 original. Dover Publications, Inc., New York, 1988.

[19] M.G. Krein, Theory of entire functions of exponential type (in Russian), Izv. Akad. Nauk. SSSR, Ser. Mat. 11 (1947), No. 4, 309-326.

[20] B.Y. Levin, Distribution of Zeros of Entire Functions, American Mathematical Society, Providence 1980. Transl. and rev. from the 1956 Russian and 1962 German editions.

[21] R.E.A.C. Paley, N. Wiener, Fourier Transforms in the Complex Domain, Amer. Math. Soc., Providence, Rhode Island, 1934.

[22] J. Rovnyak, V. Rovnyak, Sonine spaces of entire functions, J. Math. Anal. Appl., 27 (1969), 68-100.

[23] N. Sonine, Recherches sur les fonctions cylindriques et le développement des fonctions continues en séries, Math. Ann. 16 (1880), 1-80.

[24] E. C. Titchmarsh, The Theory of the Riemann-Zeta Function, 2nd ed. Edited and with a preface by D. R. Heath-Brown. Clarendon Press, Oxford 1986.

[25] H. F. Weinberger, Fourier transforms of Moebius series. Dissertation (1950), CarnegieMellon University, Pittsburgh.

Jean-François BURNOL

Université Lille 1

UFR de Mathématiques

Cité scientifique M2

F-59655 Villeneuve d'Ascq, France

E-mail : burnol@math.univ-lille1.fr 